\title{
Impact of Side-Chain Substituents on the Excited State \\ Dynamics of Perylene Diimide Amorphous Films
}

Qiu-Shi Ma ${ }^{1 \dagger^{*}}$, Cheng-Wei Ju ${ }^{2 \dagger}$, Ruihua $\mathrm{Pu}^{3}$, Wenjie Zhang ${ }^{4}$, Xian $\mathrm{Lin}^{3}$, Yihan Chen ${ }^{1 *}$, Weimin $\mathrm{Liu}^{3 *}$

${ }^{1}$ School of Resource and Environmental Engineering, Hefei University of Technology, Hefei 230009, Anhui Province, China

${ }^{2}$ College of Chemistry, Nankai University, Tianjin 300071, China

${ }^{3}$ School of Physical Science and Technology, ShanghaiTech University, Shanghai 201210, China

${ }^{4}$ Department of Physics, Shanghai University, Shanghai 200444, China

${ }^{\dagger}$ These authors contributed equally

*Email address: 2910330425@qq.com (Q. Ma), yhchen@hfut.edu.cn (Y. Chen), liuwm@shanghaitech.edu.cn (W. Liu)
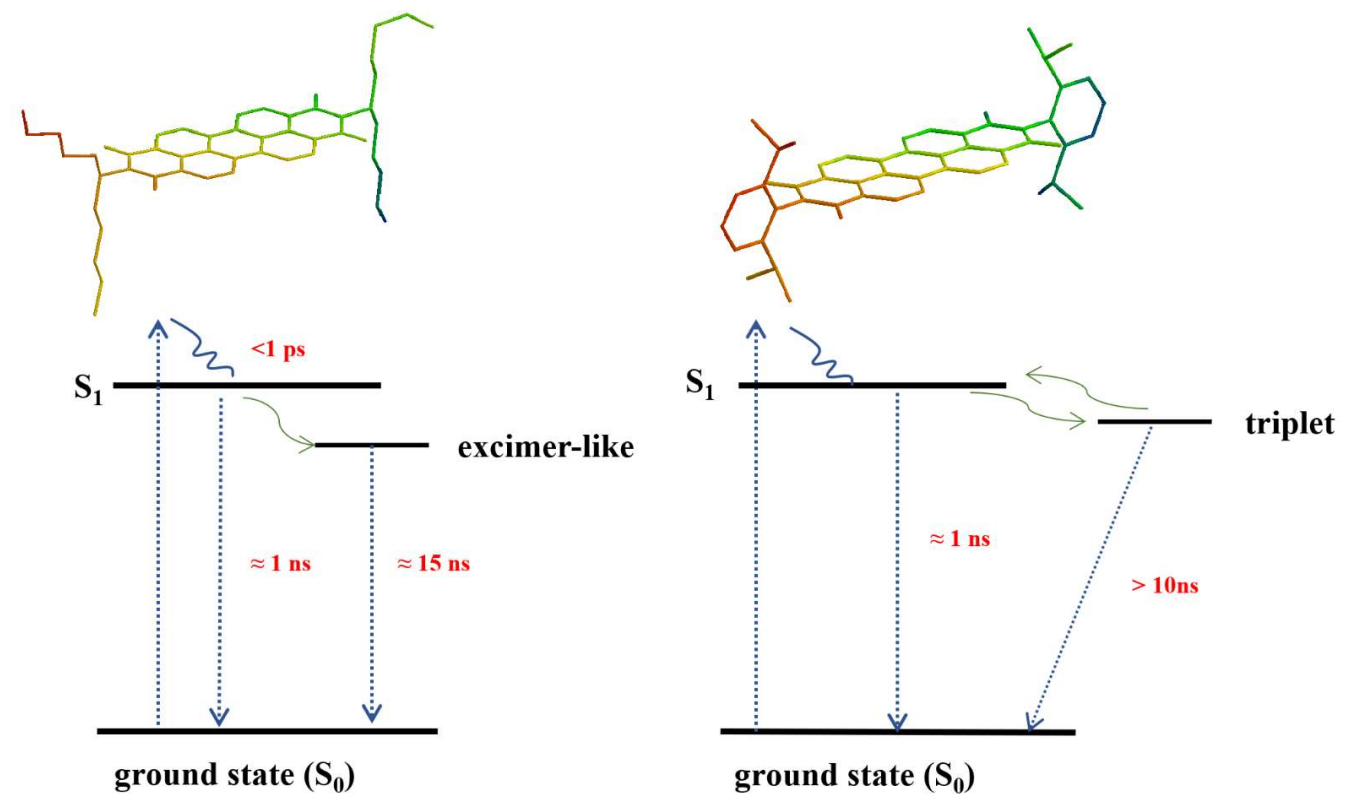


\begin{abstract}
Side-chain substitutions have important influence on the aggregation of perylene diimide (PDI), which show a great impact on their excited-state dynamics as well. Herein, by employing photoluminescence (PL), time-resolved photoluminescence (TRPL) and transient absorption (TA) spectroscopy, we investigated excited-state dynamics of two perylene diimide (PDI) derivative amorphous films, i.e. undecane-substituted PDI (PDI-1) and diisopropylphenyl-substituted PDI (PDI-2), fabricated with spin coating method. Femtosecond transient absorption spectra reveal that both films show pronounced ground state bleach (GSB) with lifetime longer than 10 ns while the relaxation of excited state absorption (ESA) has typical lifetime less than 1 ns. The significant feature of excited state decay in PDI-2 is dominated by transforming the singlet excited state into two triplet states via singlet fission, which is evidenced by the appearance of triplet state absorption. By contrast, the absence of triplet state absorption and the appearance of long-lived emission species in PDI-1 suggest that the decay of excited-like state could be dominated by the formation of excimer. Our present study reveals for the first time that the singlet fission does occur in amorphous PDI film, the study also demonstrates that side-chain substitutions have great impact on the excited-state dynamics of PDI.
\end{abstract}

Keywords: Perylene diimide derivatives, singlet fission, triplet state, excimer, molecule stacking. 


\section{Introduction}

Perylene diimides (PDIs) were employed as dye or pigments initially and had been extensively used industrial. Recently, studies on PDIs have been revived concerning their potential applications in field effect transistor ${ }^{1-2}$, photovoltaic ${ }^{3-4}$, light emitting diodes $^{5}$, degradation of organic compounds and photocatalytic anticancer treatment. ${ }^{6-10}$ The flat molecular structure in PDI that facilitates intermolecular interactions with neighboring molecules, as a result, supermolecules can be formed by molecularly stacking with side-by-side and/or head-to-tail in the solid. ${ }^{11}$ In these supermolecules, the stacking modes have great impact on their optical properties, catalytic performance, solubility, and so on. ${ }^{1,}{ }^{12}$ Moreover, substitution at imide nitrogen with various chromophores shows little effect on optical and electronic properties but can be used to tailor the aggregation and solubility of PDI molecule. ${ }^{1,13}$ Singlet exciton fission is a spin-conserved process in which a singlet excited state transfers its energy to a neighboring molecule to form two triplet excited states, each has about half of energy of the initial singlet state. ${ }^{14-18}$ In addition, the inverse process, triplet-triplet annihilation up-conversion, has also been demonstrated to occur in PDI films. ${ }^{19}$ Recent studies on singlet fission have grown rapidly because its potential for increasing the maximum efficiency of photovoltaics, by which absorbing a single photon can produce multiple excited electrons. ${ }^{14-18}$ Interestingly, the energy of triplet state of PDI was found to be approximately halfway of the lowest singlet excited state $^{14}$, which is ideal for singlet excitons to evolve into a pair of triplet excitations. 20-21 On the other hand, formation of excimer is a competitive process with singlet fission in a closely stacked organic molecule, like PDIs, which have been demonstrated to be relevant to the molecule stacks. ${ }^{22}$ Although singlet fission in various PDI films has been studied extensively in past several years, it has been noted that all studies of PDI-based singlet fission are carried out on polycrystalline films fabricated by vapor deposition method. ${ }^{14,16-18,23}$ In fact, some PDI derivatives show good solubility in weak polar solution such as chloroform and acetone ${ }^{7,24}$, and PDI films with large area and good surface morphology are able to be easily fabricated by spin coating method. This method has been widely used for film fabrication because 
of its low cost and easy to handle. In contrast to vapor deposition method, the spin-coating can only grow amorphous film. As a matter of fact, the PDI molecules can be aligned by self-assembly during spin coating process to form certain aggregation due to the strong intermolecular interaction, which is expected to show some similarity as that of polycrystalline film. So far as we learn, there is no report about the singlet exction fission and excimer formation based on amorphous PDI films. In this study, amorphous films of two PDI derivatives with different imide-substitutes are fabricated by spin coating method. By carefully adjusting spin-speed and casting time, PDI films with good surface flatness and high uniformity are obtained. By using steady absorption and PL spectra combined with transient spectroscopy, we studied the excited state dynamics of spin casting PDI films with different imide-substituents for the first time. The experimental results demonstrate that both PDI films undergo complex aggregation during spin coating process: both Jand H-aggregate are observable from the steady absorption and PL spectra. On the other hand, the difference between the two films is also obvious: the undecane-substituted PDI film shows more closely cofacial stacks, in which the H-aggregate plays more important role in the optical properties. In contrast, diisopropylphenyl-substituted PDI film shows weak interaction among PDI molecules, in which the head-to-tail alignment is preferred during the film growth, as a result the molecules are stacked with more J-aggregate. Femtosecond transient absorption (TA) spectra reveal that both films show pronounced ground state bleach (GSB) with recovery time longer than $10 \mathrm{~ns}$, and relaxation of excited state absorption (ESA) shows a typical lifetime of $\sim 1 \mathrm{~ns}$. The significant feature of excited state decay in PDI-2 is dominated by singlet exction fission that is evidenced by the appearance of triplet state absorption. In contrast, the absence of triplet state absorption and the appearance of long-lived emission species observed in TA and TRPL spectra for the PDI-1 film strongly suggest that the decay of excited state could be dominated by the formation of excimer-like state. 


\section{Experimental Section}

Materials and Characterization: Perylene-3,4,9,10 tetracarboxylic dianhydride (PTCDA), 2,6-diisopropylaniline, and imidazole were purchased from Energy Chemical and used as received. Undecan-6-amine was synthesized according to the literature. ${ }^{25}$ Analytical thin-layer chromatography (TLC) was performed on silica gel $60 \mathrm{~F}_{254}$ glass plates. The silica gel $60 \mathrm{H}$ (200-300 mesh) manufactured by Qingdao Haiyang Chemical Group Co. (China) was used for general chromatography. NMR spectra were recorded on Bruker AV 400 spectrometer at $400 \mathrm{MHz}\left({ }^{1} \mathrm{H}\right.$ NMR), 101 $\mathrm{MHz}\left({ }^{13} \mathrm{C}\right.$ NMR). The residual solvent signals were used as references for ${ }^{1} \mathrm{H}$ and ${ }^{13} \mathrm{C}$ NMR spectra and the chemical shifts were converted to the TMS scale $\left(\mathrm{CDCl}_{3}: \delta \mathrm{H}=\right.$ $7.26 \mathrm{ppm}, \delta \mathrm{C}=77.16 \mathrm{ppm})$.

Synthesis of undecane-substituted PDI (PDI-1): PTCDA (1.96 g, $5 \mathrm{mmol}$ ), undecan-6-amine (2.06 g $12 \mathrm{mmol})$ and imidazole $(10 \mathrm{~g})$ were added into a $100-\mathrm{mL}$ two-necked round-bottomed flask containing a magnetic stirring bar under $\mathrm{N}_{2}$ atmosphere. After regular stirring at $160^{\circ} \mathrm{C}$ for $4 \mathrm{~h}$, the reaction mixture was diluted with ethanol before complete cooling in order to dissolve the crystallizing imidazole. The reaction mixture was treated with added $2 \mathrm{M} \mathrm{HCl}$ solution $(100 \mathrm{~mL})$ and settled for $1 \mathrm{~h}$. The resulting precipitate was collected by suction filtration, washed with distilled water, dried under reduced pressure to give the crude product. Purification was done by flash column chromatography on silica gel (eluent: $\mathrm{PE}: \mathrm{CHCl}_{3}=1: 4$ ) afforded PDI-1 as a dark-red powder (3.21 g, 92\%). ${ }^{1} \mathrm{H}$ NMR (400 $\left.\mathrm{MHz}, \mathrm{CDCl}_{3}\right) \delta$ $8.80-8.47(\mathrm{~m}, 8 \mathrm{H}), 5.18(\mathrm{tt}, J=9.3,5.8 \mathrm{~Hz}, 2 \mathrm{H}), 2.32-2.15(\mathrm{~m}, 4 \mathrm{H}), 1.90-1.81(\mathrm{~m}$, 4H), $1.34-1.24(\mathrm{~m}, 24 \mathrm{H}), 0.83(\mathrm{t}, J=7.0 \mathrm{~Hz}, 12 \mathrm{H})$. The NMR spectrum is shown in Figure S1 of Electronic Supplementary Information (ESI). The result of the characterization is consistent with the previous work. ${ }^{26}$ The left panel in Scheme 1 shows the chemical structure of the PDI-1 molecule.

Synthesis of diisopropylphenyl-substituted PDI (PDI-2): PTCDA (1.96 g, 5 mmol), 2,6-diisopropylaniline $(2.13 \mathrm{~g}, 12 \mathrm{mmol})$ and imidazole $(10 \mathrm{~g})$ were added to a 100 -mL two-necked round-bottomed flask containing a magnetic stirring bar under $\mathrm{N}_{2}$ atmosphere. After stirring at $160^{\circ} \mathrm{C}$ for $4 \mathrm{~h}$, the reaction mixture was diluted with 
ethanol before complete cooling in order to dissolve the crystallizing imidazole. The reaction mixture was treated with added $2 \mathrm{M} \mathrm{HCl}$ solution $(100 \mathrm{~mL})$ and settled for 1 h. The resulting precipitate was collected by suction filtration, washed with distilled water, dried under reduced pressure to give the crude product. The purification by flash column chromatography on silica gel (eluent: $\mathrm{PE}: \mathrm{CHCl}_{3}=1: 4$ ) afforded PDI-2 as a dark-red powder $(2.56 \mathrm{~g}, 72 \%) .{ }^{1} \mathrm{H} \mathrm{NMR}\left(400 \mathrm{MHz}, \mathrm{CDCl}_{3}\right) \delta 8.80(\mathrm{~d}, J=8.0 \mathrm{~Hz}$, 4H), 8.75 (d, $J=8.1 \mathrm{~Hz}, 4 \mathrm{H}), 7.51$ (t, $J=7.8 \mathrm{~Hz}, 2 \mathrm{H}), 7.37$ (d, $J=7.8 \mathrm{~Hz}, 4 \mathrm{H}), 2.77$ (hept, $J=7.3 \mathrm{~Hz}, 4 \mathrm{H}), 1.19(\mathrm{~d}, J=6.8 \mathrm{~Hz}, 24 \mathrm{H}) .{ }^{13} \mathrm{C} \mathrm{NMR}\left(101 \mathrm{MHz}, \mathrm{CDCl}_{3}\right) \delta$ $163.62,145.78,135.21,132.24,130.64,130.32,129.85,126.99,124.27,123.55$, 123.49, 29.37, 24.16. The NMR spectrum is given in Figure S2 of ESI. The result of the characterization is consistent with the previous work. ${ }^{27}$ The chemical structure of PDI-2 is presented in the right panel of Scheme 1.

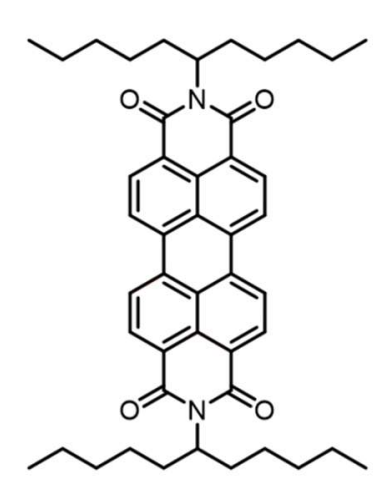

PDI-1: N,N'-di(undecan-6-yl)3,4,9,10-perylenedicarboximide

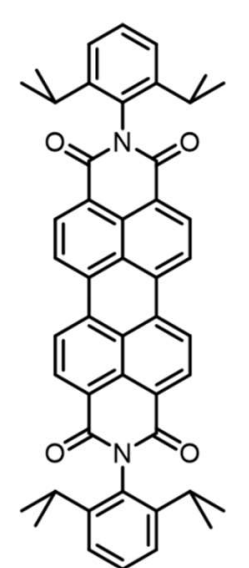

PDI-2: N,N'-di(2,6-diisopropylphenyl)3,4,9,10-perylenedicarboximide

Scheme 1. The chemical structures of two PDI derivatives

Thin Film Preparation: The PDI films were obtained by spin-coating of corresponding PDI chloroform solution with concentration $\sim 10^{-3} \mathrm{M}$ on 1-mm thick fused silica substrate. The speed of rotation is adjusted to be $30 \mathrm{rpm}$ for $5 \mathrm{~s}$ initially, then the speed turns to be about 70-80 rpm for $10 \mathrm{~s}$. The average thickness of the final film is determined to be about $300 \mathrm{~nm}$. X-ray diffraction (XRD) patterns of the films 
are shown in Figure S3 in ESI, obviously the XRD patterns of both PDI-1 and PDI-2 films are almost same as that of the substrate, fused silica, which demonstrate that the PDI spin-coating films are in amorphous phase.

Absorption and fluorescence characterization: UV-visible absorption spectra of two PDI solution and films were carried out on Shimadzu spectrometer (UV-3600). Steady and transient photoluminescence (PL) spectra for both solution and films were carried out on Edinburgh $\alpha$-1000. A picosecond time-correlated single photon counting (TCSPC) system was used for time-resolved photoluminescence (TRPL) decay measurements. The samples were excited by a 95 ps semiconductor laser (EPL-485 Edinburgh) with wavelength of $485 \mathrm{~nm}$. The instrumental response function (IRF) is better than 100 ps.

Transient absorption spectroscopy: The ultrafast spectroscopy of the two PDI films was carried out on transient absorption spectroscopy (ultrafast system, Hellos Fire Transient absorption spectrometer). Briefly, the light source was output from a commercial mode-locked Ti: sapphire laser (Coherent, Astrella tunable USP) with a central wavelength of $800 \mathrm{~nm}$, repetition rate of $1 \mathrm{kHz}$, and pulse duration of $35 \mathrm{fs}$, the incident pulse trains split into pump and probe pulses. The pump pulse was guided into an optical parametric amplifier system, which was used to tune the wavelength of the pump pulse in order to get the experimental-need wavelength beam, the pulse duration of the output from OPA was about 60 fs. The other 800 nm-beam was focused on a $1 \mathrm{~mm}$-thick sapphire in order to produce a white light continuum with pulse duration of $120 \mathrm{fs}$, which acts as probe beam in the ultrafast system. An achromatic lens was used to focus the white continuum (probe) on the spot of $0.5 \mathrm{~mm}$ in diameter, which is rough half in size of the pump beam. An optical filter was used to remove the redundant $800 \mathrm{~nm}$-beam to produce the white light ranged from $450 \mathrm{~nm}$ to $780 \mathrm{~nm}$. The pump and probe beams were focused on the surface of sample overlappingly. The pump induced the transmission change of probe beam was collected by a high sensitive charge-coupled device. In this study the pump wavelength is fixed at $485 \mathrm{~nm}$ with fluence of about $3.9 \mu \mathrm{J} / \mathrm{cm}^{2}$, which is 10 times larger than that of the probe pulse. The pump induced absorption changes of probe 
beam $(\triangle \mathrm{A})$ was used to describe the optical induced change of the PDIs films from the transient absorption spectra. $\Delta \mathrm{A}<0 \quad(>0)$ indicates a photo-induced bleaching (absorption). All measurements were performed at room temperature.

\section{Experimental Results}

The intensity normalized steady absorption and PL spectra of the two PDIs in solution and film are shown in Figure 1. The absorption and PL spectra of both PDIs in $10^{-5} \mathrm{M}$ chloroform solution show exact the same, in which absorption bands are located around 526, 489, and $458 \mathrm{~nm}$ for $0-0,0-1$, and $0-2$ vibronic bands, respectively. While the PL spectrum shows clear mirror symmetry with the absorption band, in which the $0-0,0-1$, and $0-2$ vibronic emission bands are located around 533, 573 and $620 \mathrm{~nm}$, respectively ${ }^{28}$. As mentioned above, the imide-substituent of PDI in dilute solution has negligible effect on optical properties. Through the steady absorption, it can draw a conclusion that PDIs exist in monomer form when in dilute solution ${ }^{1}$. Furthermore, the absorption and PL features in solution and films vary greatly. Comparing with the PDI maximum absorption in solution, the PDI-1 film shows blue shift of $\sim 27 \mathrm{~nm}$, indicating the dominant of $\mathrm{H}$-aggregate in film. On the other hand, the film absorption shows a flat and broad absorption band, and the onsite absorption has extended from $550 \mathrm{~nm}$ in solution to $700 \mathrm{~nm}$ in film. The PL spectrum shows the simaliar result as well: a broader and featureless emission band with large red shift $(142 \mathrm{~nm})$ relative to that of solution, which indicates J-aggregate has also existed in

the film. ${ }^{16}$ The complex optical spectra demonstrate that aggregation in PDI-1 film can't be described with either J-aggregate or H-aggregate only ${ }^{29}$. 

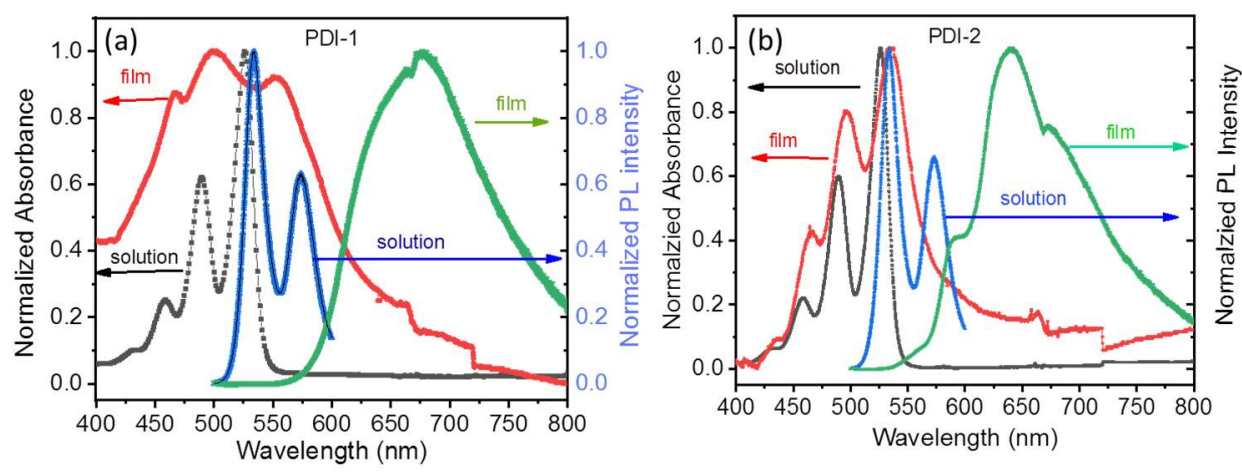

Figure 1. The intensity normalized UV-visible absorption and photoluminescence spectra of DPI-1 (a) and DPI-2 (b) in dilute $\mathrm{CHCl}_{3}$ solution and films, respectively.

Concerning the broad band absorption and featureless emission spectra with large red shift, we can infer that the molecules in PDI-1 form closely co-facial stacking, ${ }^{16}$ in which $\mathrm{H}$-aggregate plays dominated role accompanied with formation of J-aggregate during the film fabrication with spin-coating method. As illustrated in Fig. 1(b), the absorption and PL spectra of PDI-2 film show distinct features comparing with PDI-1. The PDI-2 film presents clear 0-0, 0-1 and 0-2 vibronic absorption bands, located around 535, 496 and $466 \mathrm{~nm}$, respectively, which resembles to that of the solution except for $\sim 10 \mathrm{~nm}$ red shifts. It is also noted that the PL spectrum of PDI-2 film also shows large red shift $(\sim 107 \mathrm{~nm})$ with respect to the solution. The most significant difference in PL spectra between PDI-2 and PDI-1 is that the PDI-2 film shows vibronic modulated emission resembling to the corresponding solution. Therefore we infer that the PDI-2 film undergoes head-to-tail stacking with large charge-transfer coupling in the film. ${ }^{16}$ Figure 2 presents the time-resolved PL of the two PDIs in solution and films, it can be easily seen that the solution shows monoexponential decay with typical lifetime of $\sim 3.9$ ns. In contrast, the films show multiple relaxations, the fast relaxation time with typical lifetime of $\sim 1.0 \mathrm{~ns}$, which is 4 -fold faster than that of PDI monomers in solution, indicating the presence of competing nonradiative decay processes in solid. The reduced fluorescence lifetime can be attributed to the close packing that enhances electronic coupling between molecules in the film. ${ }^{30-31} \mathrm{In}$ addition, each film displays a small, long-lived emission component that persists for 
14.8 ns for PDI-1 and 4.3 ns for PDI-2, suggesting this emission originates from a process other than PDI monomer relaxation.

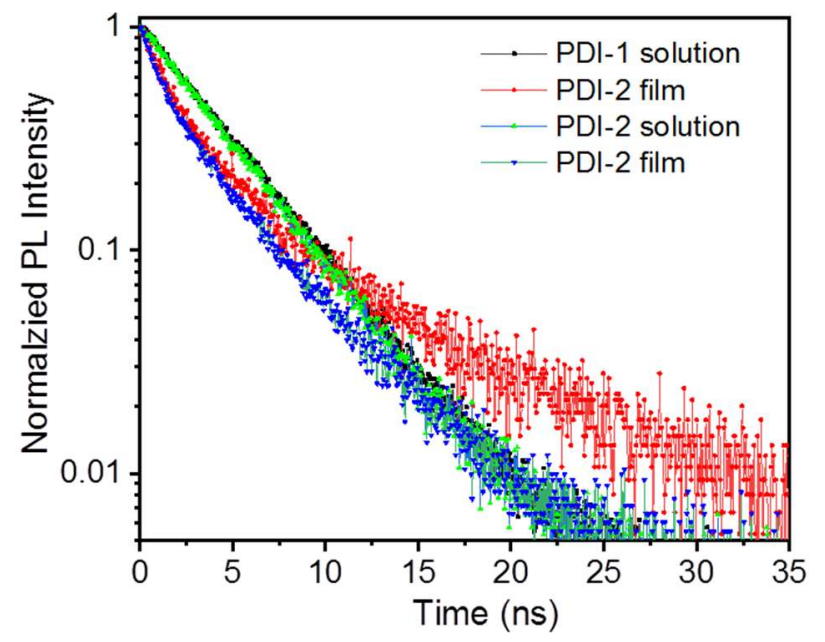

Figure 2. The intensity normalized PL spectra of PDI-1 and PDI-2 in dilute chloroform solution and films, respectively.

In order to understand the side-chain influence on the excited state dynamics, we carried out transient absorption measurement on the two films. Figure 3(a) presents the TA spectra of PDI-1 film in visible region collected at several delay times after photoexcitation at $485 \mathrm{~nm}$. The distinct features of the TA spectra are that the film behaves photoinduced bleaching centered at $558 \mathrm{~nm}$, and two photoinduced absorption peaks located around $625 \mathrm{~nm}$ and $765 \mathrm{~nm}$, respectively. The three dimensional plot of the transient absorption spectra are given in Figure S4 in ESI. The GSB at $558 \mathrm{~nm}$ corresponds to the $0-0$ vibronic transition of the film. It can be seen that the photoabsorption signal disappear completely when delay time is longer than $\Delta \mathrm{t}=1 \mathrm{~ns}$, however the photobelaching signal remains $\sim 10 \%$ of weight after $\Delta \mathrm{t}=7.5 \mathrm{~ns}$, which reveals the recovery of GSB is in the time scale much longer than the lifetime of singlet excited state. In order to learn more dynamical information of the photoexcitation, four featured wavelengths of 535, 558, 625 and $765 \mathrm{~nm}$ are selected to monitor the excited state dynamics after photoexcitation of $485 \mathrm{~nm}$. Figure 3(b) and 3(c) present the dynamical relaxations at each selected wavelength in a short time 
window (b) and long time window (c), respectively. It can be seen from Fig. 3(c) that the recovery processes of GSB at both $535 \mathrm{~nm}$ and $558 \mathrm{~nm}$ do not reach baseline up to $7.5 \mathrm{~ns}$, the limit of our experimental setup. In contrast, the relaxation of ESA at both $625 \mathrm{~nm}$ and $765 \mathrm{~nm}$ return back the to initial sate within $1 \mathrm{~ns}$. This strongly suggests that an intermediate state can be assigned to trap singlet exciton, while the ground state remains unoccupied. It can be adopted from Fig. 3(c) that the sign of the $\Delta \mathrm{A}$ at $625 \mathrm{~nm}$ changes from positive to negative occurring around $\sim 1.9 \mathrm{~ns}$, which suggests the overlapping between the photoinduced absorption of singlet state and emission of newly formed excimer-like in the film, and the formation of excimer-like state will be discussed later.
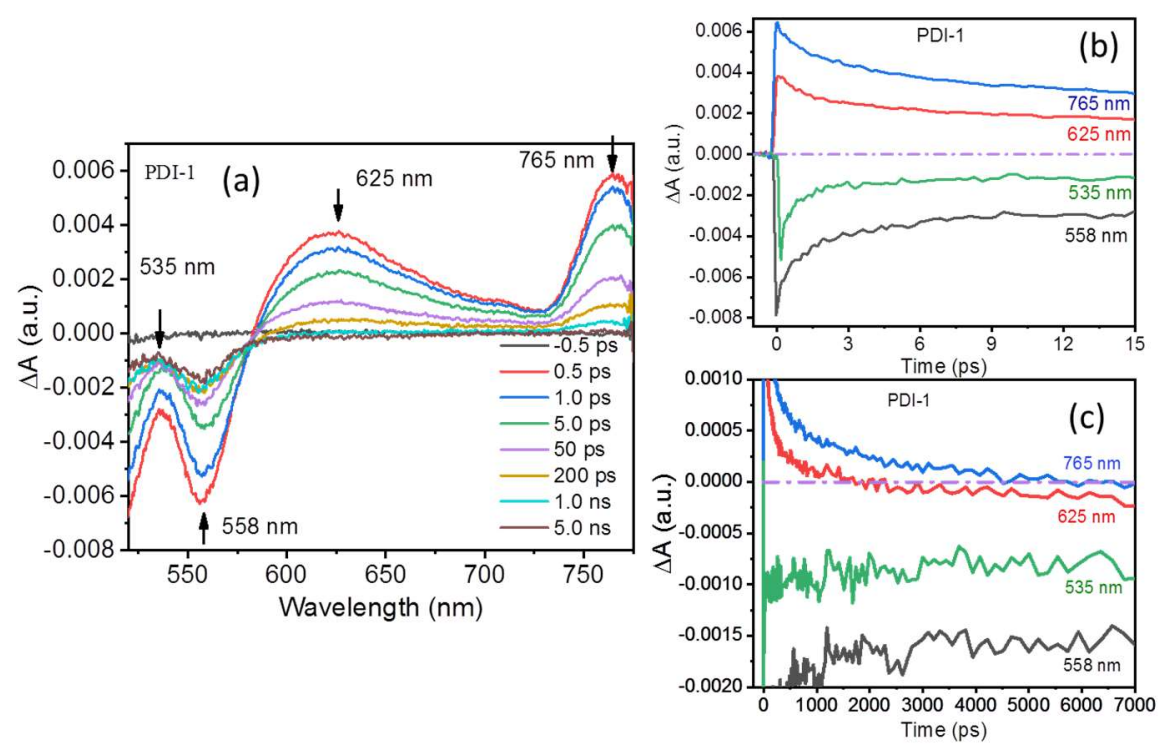

Figure 3. Transient absorption spectra of PDI-1 film after photoexcitation of $485 \mathrm{~nm}$. (a) TA spectra collected at delay times of $-0.5,0.5,1.0,5.0,50,200,1000,5000 \mathrm{ps}$, respectively. (b) Transient dynamics of PDI-1 at four selected wavelengths in short time window, and (c) Transient dynamics of PDI-1 at four selected wavelengths in long time window. 

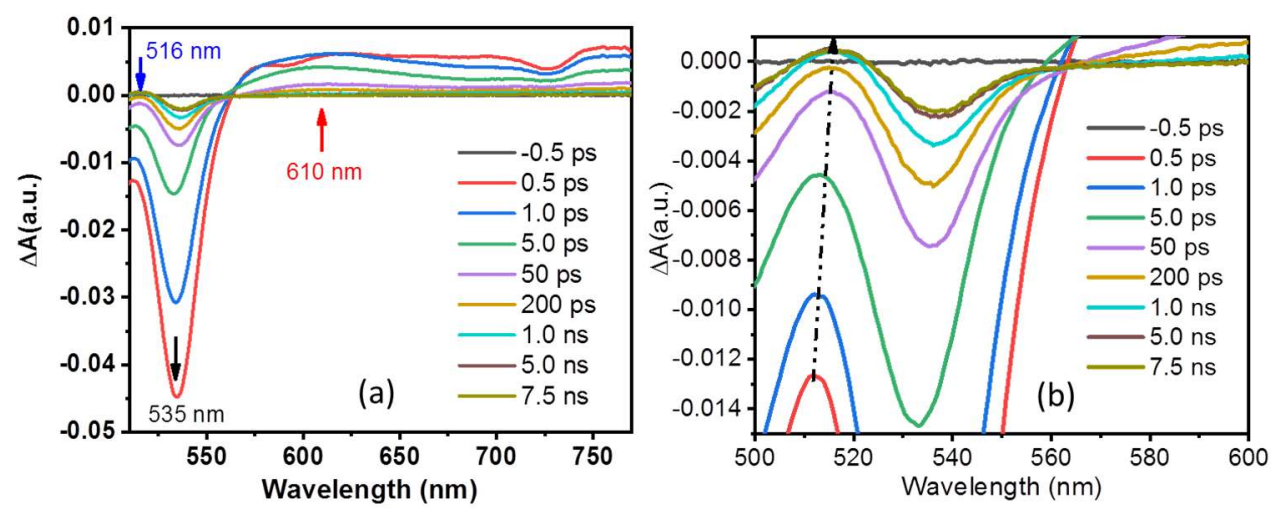

Figure 4. Transient absorption spectra of PDI-2 film after photoexcitation of $485 \mathrm{~nm}$. (a) TA spectra collected at delay times of $-0.5,0.5,1.0,5.0,50,200,1000,5000$, and $7500 \mathrm{ps,}$ respectively. (b) The enlargement of (a) around $516 \mathrm{~nm}$.

Figure 4 (a) shows the TA spectra of PDI-2 at several selected delay times. Figure S5 in ESI presents the 3D plots of the TA spectra. Different from the PDI-1, the PDI-2 shows remarkable ground state bleaching signal centered at $535 \mathrm{~nm}$ and relatively weak and flat photoinduced absorption ranged from 600 to $770 \mathrm{~nm}$. Interestingly, it is noted that there is a weak signal centered around $516 \mathrm{~nm}$, in which the signal changes from bleach in early delay time to absorption in later delay time. It is more clear that the signal shows red shift and becomes broad with delay time as illustrated in Fig. 4(b). In order to investigate the dynamical relaxation after photoexcitation of $485 \mathrm{~nm}$, we selected three featured wavelengths located respectively at 516,535 and $610 \mathrm{~nm}$ for detailed study, which is displayed in Fig. 5. Similar as that observed in PDI-1 presented in Fig. 3, the recovery time of GSB at $535 \mathrm{~nm}$ for PDI-2 is much longer than $7.5 \mathrm{~ns}$, over the limitation of our experimental setup, but the relaxation of ESA at $610 \mathrm{~nm}$ is completed within $1 \mathrm{~ns}$, this manifests the existence of intermediate state again. It is clear that the rising time of ESA is slower than that of GSB as shown in Fig. 5(b), which arises from the exciton relaxation to the lowest singlet state. In addition, the recovery of bleach signals at 516 and $535 \mathrm{~nm}$ follow almost same profile in the early stage (note: the signals at $516 \mathrm{~nm}$ and $610 \mathrm{~nm}$ are multiplied by 3 and 8 , respectively for comparison). Through Figure 5 (c), the transient dynamics of the 
zoomed-in amplitude for the three selected wavelengths, obviously, the signal of 516 $\mathrm{nm}$ is evolved from bleaching signal at early delay time into the absorption signal at later delay time, and the absorption signal does not show any obvious decay up to 7.5 ns.
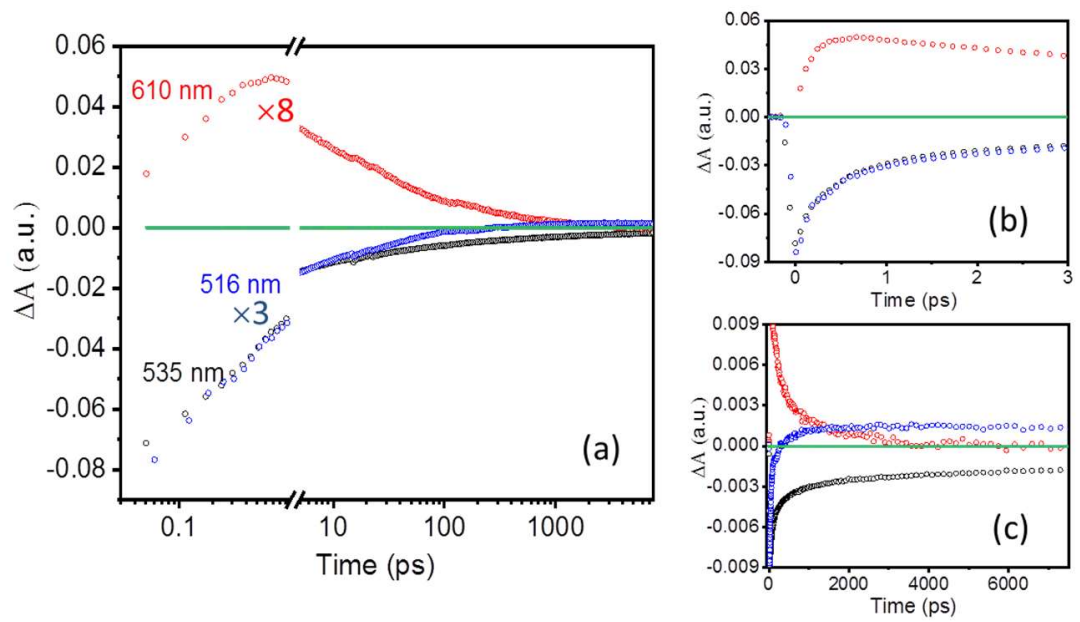

Figure 5. (a) Transient dynamics of PDI-2 film centered at $535 \mathrm{~nm}$ (black) for ground state bleach, at $516 \mathrm{~nm}$ (blue) for triplet exciton absorption, and $610 \mathrm{~nm}$ (red) for excited state absorption, respectively. (b) The transient dynamics in (a) for short time scale, and (c) Zoomed-in the amplitude of $\Delta \mathrm{A}$ in (a)

\section{Discussions}

From the TA spectra in both PDI-1 and PDI-2 films, it is strongly suggested that an intermediate state could be formed after photoexcitation. The nature of the intermediate state can be triplet state or excimer depending on the stacks of PDI molecules, which compete with each other for dissipating the excitation energy. In PDI films, long-range Columbic interactions between PDI chromophores dominate the overall shape of the absorption spectrum, and small distortion between nearest-neighbor PDIs can result in certain changes in the absorption spectra, as a result, the excited-state photodynamics of PDI films can be affected significantly. In addition, as outlined by Eaton and Le et al. ${ }^{16,22}$ short-range interaction due to charge-transfer can be either H-like or J-like depending on the degree of lateral displacement of neighboring molecules. The complex nature of excited-state 
dynamics observed in our PDI-1 and PDI-2 films arises from complex short- and long-range molecular interactions. In PDI-1 film, the undecane-substitutent at imide position tends to align PDI molecule in form of H-aggregate dominated stacks. The relaxation of ESA at $765 \mathrm{~nm}$ has lifetime of $\sim 1 \mathrm{~ns}$, while the recovery of GSB is seen to last longer than $10 \mathrm{~ns}$. In addition, there is no observable triplet absorption in the investigated wavelength, from which we can conclude that the formation of triplet state is negligible acting as the intermediate state. Besides, the relaxation of ESA at $625 \mathrm{~nm}$ is seen to develop from positive magnitude to negative one, which manifests the formation of excimer-like state after photoexcitation. ${ }^{28}$ The magnitude of $\Delta \mathrm{A}$ at $625 \mathrm{~nm}$ is resulted from the superposition of excited state absorption and excimer-like emission. The lifetime of the excimer in PDI-1 film is about $14.8 \mathrm{~ns}$ as determined from TRPL measurement shown in Fig. 2, which is comparable to the reported excimer lifetime in PDI solutions. ${ }^{32-33}$ In order to confirm the excimer formation dynamics in PDI-1 film, we have measured transient fluorescence spectra, which are illustrated in Fig. 6. Figure 6(a) and 6(b) present the two-dimensional transient fluorescence contour map and the transient photoluminescence spectra collected at several delay times, respectively. In thin film, the co-facially stacked PDI aggregates generally have a widen absorption and large red-shift fluorescence bands with significantly longer decay time than that of solution mentioned above. In addition, with the evidence of the TRPL spectra that show red-shift and broader bandwidth with delay time shown in Fig. 6. Hence, it is reasonable to draw a conclusion that the intermediate state in PDI-1 can be ascribed to the "excimer-like" state. ${ }^{34-35}$ As the formation of excimer can suppress significantly the formation of triplet state by singlet fission process, therefore it is predictable no triplet absorption can be detected in the TA spectrum. ${ }^{31,36}$ 

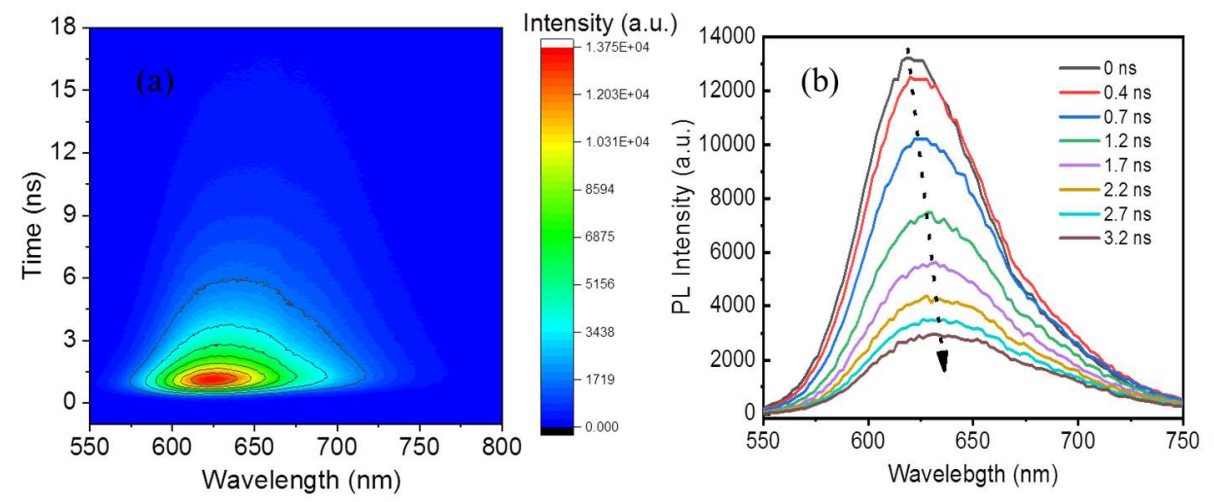

Figure 6 Two dimensional transient photoluminescence contour map (a) and transient fluorescence spectra collected at several delay times (b) for the PDI-1 film under photoexcitation of $485 \mathrm{~nm}$.

In contrast, diisopropylphenyl-substituted PDI hinders the molecule stack cofacially, and tend to align in head-to-tail stack, and form a J-aggregate like film. ${ }^{1}$ Accompanied with the relaxation of the ESA in PDI-2 film, a new absorption band centered on 516 nm can be clearly seen. The character of the new absorption band is seen to show red-shift and becomes broader with delay time, which strongly suggests the intermediate state is a triplet state in nature. In other word, the excitation of PDI-2 film undergoes singlet exciton fission to produce triplet excitons after photoexcitation. As the weak intermolecular interaction can suppresses the formation of excimer in this kind of PDI film, so that the triplet state absorption can be clearly identified from the TA spectra. By recalling the TRPL spectrum of PDI-2 presented in Fig. 2, the slow emission process with time constant of 4.3 ns can be assigned as the delayed PL emission, in which the delayed singlet emission arises from the triplet-triplet annihilation to convert back into singlet state ${ }^{19}$. It should be mentioned that the singlet fission rate in our case is hard to estimate due to the overlapping between GSB and triplet state absorption at $516 \mathrm{~nm}$. Finally, it should be mentioned that the kinetics of TA and TCSPC data display significant difference for the two PDI films, the TA data show an ultrafast component with typical time constant of $\sim 1$ ps. The marked difference is due to the two experiments are not performed under identical conditions. 
Much higher excitation densities are used in TA measurement, which leads to singlet-singlet exciton annihilation. ${ }^{19,32-33}$ The subpicosecond component observed in TA data of PDI films can be interpreted as the singlet-singlet annihilation accelerating decay dynamics.

In conclusion, two PDI films, i.e. undecane- and diisopropylphenyl-substitutent of PDI at imide position, were fabricated with spin coating technique. Two films show complex absorption and PL spectra due to the complex short- and long-range molecular interactions. In general, the undecane-substituted PDI film shows more H-aggregate like characteristic, while the diisopropylphenyl-substituted PDI film has more J-aggregate like stacking. The TA spectra of the two films reveal that the excited-state dynamics of two films are dominated by different intermediate states. For PDI-1 film, photoexcitation leads to the formation of excimer-like state that dominates the dynamics of excited states. In contrast, the excited state dynamics in PDI-2 is dominated by singlet fission process, in which triplet states are produced. The time resolved photoluminescence spectra show good agreement with the TA measurement. Our present study not only extends the understanding of singlet fission in PDI-based film, which provides a low cost way to fabricate PDI-based photovoltaic and optoelectronic devices concerning the singlet fission process, the study also sheds light on the understanding the role of imide-substituents on the molecular packing, solid state fluorescence and excited state dynamics of organic molecules, which also paves the way for tailoring the stacking style of PDI molecules by attaching certain subgroup substitutions on the main frame.

\section{Acknowledgement}

We are grateful to Dr. Dongbing Zhao and Dr. Liping Lv for their helpful discussion. This work was supported by the National Natural Science Foundation of China (NSFC, Nos. 11774233, 11674213). 


\section{References:}

1. Huang, C.; Barlow, S.; Marder, S. R., Perylene-3,4,9,10-Tetracarboxylic Acid Diimides: Synthesis, Physical Properties, and Use in Organic Electronics. J Org Chem 2011, 76, 2386-407.

2. Cheng, H.; Huai, J.; Cao, L.; Li, Z., Novel Self-Assembled Phosphonic Acids Monolayers Applied in N-Channel Perylene Diimide (Pdi) Organic Field Effect Transistors. Applied Surface Science 2016, 378, 545-551.

3. Tamai, Y.; Fan, Y.; Kim, V. O.; Ziabrev, K.; Rao, A.; Barlow, S.; Marder, S. R.; Friend, R. H.; Menke, S. M., Ultrafast Long-Range Charge Separation in Nonfullerene Organic Solar Cells. ACS Nano 2017, 11, 12473-12481.

4. Hartnett, P. E., et al., Slip-Stacked Perylenediimides as an Alternative Strategy for High Efficiency Nonfullerene Acceptors in Organic Photovoltaics. J Am Chem Soc 2014, 136, 16345-56.

5. Qin, Y.; Li, G.; Qi, T.; Huang, H., Aromatic Imide/Amide-Based Organic Small-Molecule Emitters for Organic Light-Emitting Diodes. Materials Chemistry Frontiers 2020, 4, 1554-1568.

6. Wei, W.; Liu, D.; Wei, Z.; Zhu, Y., Short-Range П-П Stacking Assembly on P25 Tio2 Nanoparticles for Enhanced Visible-Light Photocatalysis. ACS Catalysis 2016, 7, 652-663.

7. Wang, J.; Shi, W.; Liu, D.; Zhang, Z.; Zhu, Y.; Wang, D., Supramolecular Organic Nanofibers with Highly Efficient and Stable Visible Light Photooxidation Performance. Applied Catalysis B: Environmental 2017, 202, 289-297.

8. Wang, J.; Liu, D.; Zhu, Y.; Zhou, S.; Guan, S., Supramolecular Packing Dominant Photocatalytic Oxidation and Anticancer Performance of Pdi. Applied Catalysis B: Environmental 2018, 231, 251-261.

9. Cai, T.; Zeng, W.; Liu, Y.; Wang, L.; Dong, W.; Chen, H.; Xia, X., A Promising Inorganic-Organic Z-Scheme Photocatalyst Ag3po4/Pdi Supermolecule with Enhanced Photoactivity and Photostability for Environmental Remediation. Applied Catalysis B: Environmental 2020, 263.

10. Nattestad, A.; Mozer, A. J.; Fischer, M. K.; Cheng, Y. B.; Mishra, A.; Bauerle, P.; Bach, U., Highly Efficient Photocathodes for Dye-Sensitized Tandem Solar Cells. Nat Mater 2010, 9, 31-5.

11. Ghosh, S.; Li, X. Q.; Stepanenko, V.; Wurthner, F., Control of H- and J-Type Pi Stacking by Peripheral Alkyl Chains and Self-Sorting Phenomena in Perylene Bisimide Homo- and Heteroaggregates. Chemistry 2008, 14, 11343-57.

12. Naqvi, S.; Kumar, M.; Kumar, R., Facile Synthesis and Evaluation of Electron Transport and Photophysical Properties of Photoluminescent Pdi Derivatives. ACS Omega 2019, 4, 19735-19745.

13. Pagoaga, B.; Mongin, O.; Caselli, M.; Vanossi, D.; Momicchioli, F.; Blanchard-Desce, M.; Lemercier, G.; Hoffmann, N.; Ponterini, G., Optical and Photophysical Properties of Anisole- and Cyanobenzene-Substituted Perylene Diimides. Phys Chem Chem Phys 2016, 18, 4924-41.

14. Yaroslav V. Aulin, K. M. F., Deniz Gunbas, Rajeev K; Dubey, W. F. J., and Ferdinand Grozema, Morphology Independent Efficient Singlet Exciton Fission in Pdi Films ChemPlusChem 2017, 83, 230-238.

15. Kamat, W. E. F. a. P. V., Photochemistry of 3,4,9,10-Perylenetetracarboxylic Dlanhydrlde Dyes. 3. Singlet and

Triplet Excited-State Properties of the Bls(2,5-Di-Ferf -Butylphenyl)Imide Derivative. J. Phys. Chem. 1987, 91, 6373-6380.

16. Le, A. K.; Bender, J. A.; Arias, D. H.; Cotton, D. E.; Johnson, J. C.; Roberts, S. T., Singlet Fission Involves an Interplay between Energetic Driving Force and Electronic Coupling in Perylenediimide Films. J Am Chem Soc 2018, 140, 814-826.

17. Felter, K. M.; Grozema, F. C., Singlet Fission in Crystalline Organic Materials: Recent Insights and 
Future Directions. J Phys Chem Lett 2019, 10, 7208-7214.

18. Felter, K. M.; Dubey, R. K.; Grozema, F. C., Relation between Molecular Packing and Singlet Fission in Thin Films of Brominated Perylenediimides. J Chem Phys 2019, 151, 094301.

19. Le, A. K.; Bender, J. A.; Roberts, S. T., Slow Singlet Fission Observed in a Polycrystalline Perylenediimide Thin Film. J Phys Chem Lett 2016, 7, 4922-4928.

20. Tayebjee, M. J.; McCamey, D. R.; Schmidt, T. W., Beyond Shockley-Queisser: Molecular Approaches to High-Efficiency Photovoltaics. J Phys Chem Lett 2015, 6, 2367-78.

21. Tayebjee, M. J. Y.; Gray-Weale, A. A.; Schmidt, T. W., Thermodynamic Limit of Exciton Fission Solar Cell Efficiency. The Journal of Physical Chemistry Letters 2012, 3, 2749-2754.

22. Lefler, K. M.; Brown, K. E.; Salamant, W. A.; Dyar, S. M.; Knowles, K. E.; Wasielewski, M. R., Triplet State Formation in Photoexcited Slip-Stacked Perylene-3,4:9,10-Bis(Dicarboximide) Dimers on a Xanthene Scaffold. J Phys Chem A 2013, 117, 10333-45.

23. Wang, L.; Bai, S.; Wu, Y.; Liu, Y.; Yao, J.; Fu, H., Revealing the Nature of Singlet Fission under the Veil of Internal Conversion. Angew Chem Int Ed Engl 2020, 59, 2003-2007.

24. Boobalan, G.; Imran, P. M.; Ramkumar, S. G.; Nagarajan, S., Fabrication of Luminescent Perylene Bisimide Nanorods. Journal of Luminescence 2014, 146, 387-393.

25. Yano, Y.; Wang, F.; Mitoma, N.; Miyauchi, Y.; Ito, H.; Itami, K., Step-Growth Annulative Pi-Extension Polymerization for Synthesis of Cove-Type Graphene Nanoribbons. J Am Chem Soc 2020, 142, 1686-1691.

26. Xu, X.; Austin, A.; Mylon, S. E.; Plenge, J.; Szarko, J. M., Improving the Quantum Yields of Perylene Diimide Aggregates by Increasing Molecular Hydrophobicity in Polar Media. Chemphyschem 2017, 18, 2430-2441.

27. Regar, R.; Mishra, R.; Mondal, P. K.; Sankar, J., Metal-Free Annulation at the Ortho- and Bay-Positions of Perylene Bisimide Leading to Lateral Pi-Extension with Strong Nir Absorption. J Org Chem 2018, 83, 9547-9552.

28. Song, H.; Zhao, H.; Guo, Y.; Philip, A. M.; Guo, Q.; Hariharan, M.; Xia, A., Distinct Excited-State Dynamics of near-Orthogonal Perylenimide Dimer: Conformational Planarization Versus Symmetry Breaking Charge Transfer. The Journal of Physical Chemistry C 2019, 124, 237-245.

29. Oleson, A., et al., Perylene Diimide-Based $\mathrm{Hj}$ - and $\mathrm{Hj}$-Aggregates: The Prospect of Exciton Band Shape Engineering in Organic Materials. The Journal of Physical Chemistry C 2019, 123, 20567-20578.

30. Dover, C. B.; Gallaher, J. K.; Frazer, L.; Tapping, P. C.; Petty, A. J., 2nd; Crossley, M. J.; Anthony, J. E.; Kee, T. W.; Schmidt, T. W., Endothermic Singlet Fission Is Hindered by Excimer Formation. Nat Chem 2018, 10, 305-310.

31. Kaufmann, C.; Kim, W.; Nowak-Krol, A.; Hong, Y.; Kim, D.; Wurthner, F., Ultrafast Exciton Delocalization, Localization, and Excimer Formation Dynamics in a Highly Defined Perylene Bisimide Quadruple Pi-Stack. J Am Chem Soc 2018, 140, 4253-4258.

32. Chen, W.; Zhu, Z.; Yin, C.; Li, Y.; Liu, Y.; Zhang, Y.; Fan, Y.; Fan, X., Water-Induced Formation of a Chiral Phenylalanine Derivative Supramolecule. Phys Chem Chem Phys 2018, 20, 4144-4148.

33. Son, M.; Park, K. H.; Shao, C.; Wurthner, F.; Kim, D., Spectroscopic Demonstration of Exciton Dynamics and Excimer Formation in a Sterically Controlled Perylene Bisimide Dimer Aggregate. J Phys Chem Lett 2014, 5, 3601-7.

34. Raj, M. R.; Margabandu, R.; Mangalaraja, R. V.; Anandan, S., Influence of Imide-Substituents on the H-Type Aggregates of Perylene Diimides Bearing Cetyloxy Side-Chains at Bay Positions. Soft Matter 2017, 13, 9179-9191. 
35. Roberts, S. T.; McAnally, R. E.; Mastron, J. N.; Webber, D. H.; Whited, M. T.; Brutchey, R. L.; Thompson, M. E.; Bradforth, S. E., Efficient Singlet Fission Discovered in a Disordered Acene Film. J Am Chem Soc 2012, 134, 6388-400.

36. Sung, J.; Nowak-Krol, A.; Schlosser, F.; Fimmel, B.; Kim, W.; Kim, D.; Wurthner, F., Direct Observation of Excimer-Mediated Intramolecular Electron Transfer in a Cofacially-Stacked Perylene Bisimide Pair. J Am Chem Soc 2016, 138, 9029-32. 